\title{
CARBONO ORGÂNICO E ATRIBUTOS QUÍMICOS DO SOLO EM ÁREAS FLORESTAIS ${ }^{1}$
}

\author{
Álvaro Luiz Mafra², Sulamita de Fátima Figueiredo Guedes², Osmar Klauberg Filho², Júlio César Pires \\ Santos $^{2}$, Jaime Antônio de Almeida² e Jaqueline Dalla Rosa ${ }^{3}$
}

\begin{abstract}
RESUMO - O objetivo deste estudo foi avaliar teores de carbono orgânico e atributos químicos do solo em áreas localizadas em Campo Belo do Sul, SC, nos seguintes usos da terra: campo nativo (CN); floresta de pinus (Pinus taeda) com 12 anos (P12); floresta de pinus com 20 anos (P20); reflorestamento de araucária (Araucaria angustifolia) com 18 anos (A18); e mata nativa de araucária (MN). O solo foi amostrado de forma sistemática, com oito repetições, coletando-se as camadas de $0,0-0,05 ; 0,05-0,1 ; 0,1-0,2$; e $0,2-0,4 \mathrm{~m}$. Os reflorestamentos com pinus e araucária mantiveram os estoques de C orgânico na camada do solo de 0,0-0,4 m em níveis equivalentes aos de mata e campo, totalizando de 12,5 a $14,2 \mathrm{~kg} \mathrm{~m}^{-2}$. A acidez do solo e os teores de P disponível foram maiores, na média das camadas analisadas, no tratamento P20. Entre os nutrientes, verificaram-se teores baixos a médios de $\mathrm{P}$ disponível e de $\mathrm{K}, \mathrm{Ca}$ e Mg trocável.
\end{abstract}

Palavras-chave: Acidez do solo, nutrientes e reflorestamento.

\section{ORGANIC CARBON AND SOIL CHEMICAL ATTRIBUTES IN FOREST AREAS}

\begin{abstract}
The objective of this work was to evaluate organic carbon contents and soil chemical attributes in forest areas in Campo Belo do Sul, Santa Catarina, Brazil, with the following land use: native grassland (NG); 12-year-old loblolly pine (Pinus taeda) reforestation (P12); 20-year-old loblolly pine reforestation (P20); 18-year-old araucaria (Araucaria angustifolia) reforestation (A18); and native forest with araucaria $(N F)$. The soil samples were systematically collected with eight replicates, in the layers of 0.0-0.05; 0.050.1; 0.1-0.2 and 0.2-0.4 m. The pinus and araucaria reforestations showed organic C stocks similar to those in native forest and grassland areas, reaching 12.5 to $14.2 \mathrm{~kg} \mathrm{~m}^{-2}$ in the layer of 0.0-0.4 $\mathrm{m}$ depth. Soil acidity and available $P$ contents were highest, in the mean of the analyzed soil layers, under the P2O treatment. Soil nutrient status ranged from low to medium for available $P$ and exchangeable $K, C a$ and $M g$.
\end{abstract}

Keywords: Carbon sequestration, nutrients and reforestation.

\section{INTRODUÇÃ̃O}

O plantio de espécies florestais, especialmente de Pinus taeda e de araucária, para produção de madeira e celulose é uma das atividades agroflorestais que mais têm crescido nos últimos anos na região do Planalto Sul catarinense. A expansão desses reflorestamentos ocorre muitas vezes em áreas de campos naturais, que consistem num ecossistema com rica biodiversidade ainda pouco conhecida. No entanto, essas espécies florestais apresentam boa adaptação edafoclimática e ampla gama de utilização, o que pode diminuir a pressão pela exploração das florestas nativas, contribuindo para a conservação dessas áreas (FERRAZ e MOTTA, 2000).

\footnotetext{
${ }^{1}$ Recebido em 16.01.2007 e aceito para publicação em 20.02.2008.

${ }^{2}$ Centro de Ciências Agroveterinárias, Departamento de Solos e Recursos Naturais da Universidade do Estado de Santa Catarina (UDESC). E-mail: <a2alm@ cav.udesc.br>.

${ }^{3}$ Mestre em Manejo do Solo, UDESC Doutoranda em Solos e Nutrição de Plantas, ESALQ/USP.
} 
Do ponto de vista edáfico, as alterações no uso da terra têm estreita relação com a dinâmica da matéria orgânica e com o ciclo biogeoquímico dos elementos, modificando a capacidade produtiva desses ambientes, naturalmente ligados a condições de solos ácidos e intemperizados. Nesses locais, o suprimento de nutrientes nas formações vegetais é dependente, em grande parte, da contribuição das substâncias orgânicas, uma vez que a reserva de elementos provenientes dos minerais é reduzida (BROWN et al., 1994).

Deve-se considerar que a dinâmica do carbono, juntamente com os nutrientes minerais, é influenciada por fatores como clima, tipo de solo, cobertura vegetal e práticas de manejo. No caso das espécies florestais, observa-se maior capacidade de ciclagem de nutrientes que as plantas de ciclo anual, em virtude do sistema radicular permanente e profundo que absorve elementos de camadas subsuperficiais, retornando-os à superfície pela deposição de serapilheira (HAAG, 1985).

Dessa forma, há crescente interesse no estudo do comportamento dos solos quanto à sua capacidade de armazenar carbono e nutrientes, em resposta às diversas condições de manejo adotadas. A avaliação dessas mudanças auxiliaria a compreensão das alterações no ambiente advindas do reflorestamento, contribuindo para o planejamento do manejo florestal. Assim, o objetivo deste trabalho foi quantificar os teores e estoques de carbono orgânico, bem como caracterizar a composição química do solo sob diferentes usos florestais.

\section{MATERIAL E MÉTODOS}

O trabalho foi conduzido em Campo Belo do Sul, SC, no Planalto dos Campos Gerais, sendo o solo na região um Nitossolo Háplico. A vegetação primária era mata de araucária, com relevo suave-ondulado e ondulado. O clima é mesotérmico úmido com verão ameno, Cfb, segundo a classificação de Köppen. A altitude varia de 950 a $1.000 \mathrm{~m}$. A temperatura média anual é de $15,6^{\circ} \mathrm{C}$ e a precipitação média anual, cerca de $1.400 \mathrm{~mm}$, bem distribuída ao longo do ano (SANTA CATARINA, 1986).

Os tratamentos constaram de cinco sistemas de uso da terra: a) campo nativo (CN) sem pastejo desde 1993, roçado anualmente em março; b) floresta de pinus (Pinus taeda) com 12 anos de idade (P12), plantada no espaçamento de $1,6 \times 2,5 \mathrm{~m}$, em área de campo nativo, após subsolagem e nivelamento com enxada rotativa, sem calagem nem adubação; c) floresta de pinus com 20 anos de idade (P20) nas mesmas condições da floresta anterior; d) reflorestamento de araucária (Araucaria angustifolia) com 18 anos de idade (A18) plantado em área de campo nativo até 1980 , quando foi implantada a pastagem de festuca, aplicando-se $8 \mathrm{Mg} \mathrm{ha}^{-1}$ de calcário dolomítico distribuído mecanicamente a lanço, metade da dose antes da aração e metade antes da gradagem - o plantio de araucária foi feito em 1985, em solo subsolado e nivelado com enxada rotativa, no espaçamento de $1,6 \times 2,5 \mathrm{~m}$; e) mata nativa com araucária $(\mathrm{MN})$, sem entrada de bovinos no sub-bosque desde 1993.

As áreas foram selecionadas num raio máximo de distância de $2 \mathrm{~km}$ entre elas, de forma a apresentar características edáficas e de relevo semelhantes, o que foi avaliado em cortes nas laterais das estradas e com base em tradagens efetuadas em transectos paralelos, observando-se profundidade dos horizontes, textura e cor do solo. As amostras de solo foram coletadas de forma sistemática, em quatro pontos eqüidistantes de $10 \mathrm{~m}$, distribuídos em dois transectos paralelos entre si, totalizando oito pontos amostrais, numa área experimental de $40 \times 20 \mathrm{~m}$ em cada forma de uso da terra. As profundidades de amostragem foram: 0,0-0,05; $0,5-0,1 ; 0,1-0,2 ;$ e $0,2-0,4 \mathrm{~m}$, com coleta de solo ao redor de uma trincheira com cerca de $0,4 \mathrm{~m}$ de largura, eliminando-se a serapilheira da superfície.

A composição química do solo compreendeu a determinação de carbono orgânico, $\mathrm{pH}$ em água, $\mathrm{pH}$ em $\mathrm{CaCl}_{2}$, pH SMP, teores de P disponível, $\mathrm{K}, \mathrm{Ca}, \mathrm{Mg}$ e Al trocável (TEDESCO et al., 1995). Os estoques de carbono foram computados de forma ponderada, considerando-se os teores e as respectivas densidades do solo em cada camada, determinadas pelo método do anel volumétrico.

Os resultados foram submetidos à análise de normalidade pelo teste de Shapiro-Wilk (SHAPIRO e WILK, 1965). A análise de variância foi feita com o uso do teste F, considerando-se delineamento inteiramente casualizado com parcelas subdivididas. As parcelas corresponderam aos cinco sistemas de uso da terra e as subparcelas, às quatro profundidades de coleta. A comparação de médias em cada camada foi realizada por Tukey a $5 \%$ de probabilidade. $O$ fator profundidade de amostragem foi avaliado por análise de regressão, detalhada para cada sistema de uso do

\section{R. Árvore, Viçosa-MG, v.32, n.2, p.217-224, 2008}

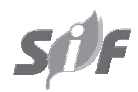


solo, quando houve interação significativa $(\mathrm{p}=0,05)$ entre sistemas e profundidades. As relações entre os atributos edáficos foram determinadas por análise de correlação de Pearson.

\section{RESULTADOS E DISCUSSÃO}

O teor de carbono orgânico no solo variou de 23 a $56 \mathrm{~g} \mathrm{~kg}^{-1}$, com maior concentração na camada de 0,0 a $0,05 \mathrm{~m}$ na mata nativa $(\mathrm{MN})$ e no campo nativo $(\mathrm{CN})$, em relação aos demais tratamentos (Tabela 1). Já em subsuperfície de 0,2 a 0,4 m, se verificou que a floresta de pinus com 20 anos de idade (P20) apresentou maior teor de carbono em relação aos demais sistemas. $\mathrm{Na}$ média das profundidades, o tratamento P20 teve o maior teor de carbono, não diferindo do campo nativo (CN). A substituição do ecossistema campo por reflorestamento de pinus aumentou o conteúdo de carbono do solo após 20 anos, conforme constatado no Paraná, com ${ }^{13} \mathrm{C}$ como traçador isotópico (LIMA et al., 1995). Na mata nativa (MN), o teor de carbono diminuiu em profundidade com o ajuste quadrático. Nas demais áreas, o decréscimo foi linear. A adição de material orgânico proveniente, principalmente, da serapilheira é responsável pelo acúmulo de carbono na camada superficial do solo, à medida que vai sendo humificada. Tal contribuição em florestas subtropicais com araucária variou de 5,9 $\mathrm{Mg} \mathrm{ha}^{-1}$ (FERNANDES e BACKES, 1998) a 7,7 $\mathrm{Mg} \mathrm{ha}^{-1}$ de matéria seca ao ano (FIGUEIREDO FILHO et al., 2003). No caso de reflorestamentos, o acúmulo anual de serapilheira de Pinus taeda com 15 anos de idade, na região de Ponta Grossa, PR, variou de 6,8 a $8,5 \mathrm{Mg} \mathrm{ha}^{-1}$ de massa seca (WISNIEWSKI e REISSMANN, 1996). O teor de carbono orgânico no solo é dependente das taxas de adição e decomposição da biomassa. Em florestas de pinus da região Sul do Brasil, tem-se observado maior acúmulo de resíduos orgânicos na superfície do solo em relação às florestas nativas em razão, principalmente, da dificuldade de decomposição da fitomassa (TREVISAN et al., 1987).

Os estoques de carbono orgânico de 0,0 a 0,4 m de profundidade totalizaram de 12,5 a $14,2 \mathrm{~kg} \mathrm{~m}^{-2}$, com maior estoque no reflorestamento de pinus com 20 anos de idade, não diferindo do campo nativo e da mata nativa (Tabela 1). Na camada de 0,0 a $0,05 \mathrm{~m}$, o solo sob mata nativa apresentou maior estoque. Em subsuperfície de 0,2 a 0,4 m, os estoques foram superiores no pinus com 20 anos de idade e no campo nativo. Balbinot et al. (2003), ao avaliarem o estoque de C em plantio de Pinus taeda com 5 anos de idade em Cambará do Sul, RS, observaram quantidades de 14,8 e $22,8 \mathrm{~kg} \mathrm{~m}^{-2}$ nas camadas de 0,0 a 0,4 e 0,0 a 1,0 $\mathrm{m}$ de profundidade, respectivamente. Lima et al. (1995), avaliando a substituição do campo natural por plantio de Pinus elliottii em quatro regiões do Paraná, constataram acréscimo de $11,3 \mathrm{Mg} \mathrm{ha}^{-1}$ de $\mathrm{C}$ no pinus em relação ao campo após 20 anos, na camada de 0,0 a $0,8 \mathrm{~m}$ de profundidade do solo, atingindo estoque de $\mathrm{C}$ de $21,4 \mathrm{~kg} \mathrm{~m}^{-2}$. No caso de sistemas florestais, além do armazenamento de $\mathrm{C}$ no solo, pode-se ter considerável estoque desse elemento na parte aérea das árvores, o que, ao longo do tempo, pode interagir com o solo pela decomposição da serapilheira. Num estudo realizado na região de Cambará do Sul, RS, verificou-se que a produtividade média do Pinus taeda aos 28 anos de idade foi de $911 \mathrm{~m}^{3} \mathrm{ha}^{-1}$, o que representa considerável estoque de carbono (MAINARDI et al., 1996). Em outro estudo na região de Curitiba, PR, verificou-se acúmulo de 60,9 Mg ha $^{-1}$ de $\mathrm{C}$ na biomassa da bracatinga após oito anos (FEISTAUER et al., 2004). Esses resultados evidenciam o potencial das florestas em armazenar carbono no solo e na fitomassa, o que seria conveniente termos econômicos (PAIXÃO et al., 2006) e ambientais, especialmente pelo seu papel na conservação do solo e da água (FEARNSIDE, 2000).

$\mathrm{O}$ pH em água variou de 4,5 a 5,3, com menores valores nos plantios de pinus P12 e P20, em todas as camadas (Tabela 2). No reflorestamento de araucária, o pH do solo foi influenciado pela calagem efetuada em 1980. Para opH em $\mathrm{CaCl}_{2}$, os menores valores ocorreram no $\mathrm{P} 20$, em todas as camadas. $\mathrm{O}$ pH em SMP diferenciou os plantios de pinus, com menores valores de índice SMP no P20 em relação ao P12, em todas as camadas, indicando incremento da acidez potencial do solo com aumento da idade do reflorestamento. Não houve variação do pH em água e SMP em profundidade, sendo evidenciados baixos coeficientes de determinação das equações para pH em sal (Tabela 2). Os efeitos da vegetação sobre a acidez do solo foram estudados por Silva (1988) na região Sul da Bahia, não havendo diferença no pH do solo sob Pinus caribaea var. hondurensis em relação à mata nativa. Em Latossolo argiloso da região de Uberlândia, MG, verificou-se que

R. Árvore, Viçosa-MG, v.32, n.2, p.217-224, 2008 
o cultivo de Pinus caribaea, embora não tenha alterado o pH do solo, reduziu de 0,2 a 0,6 unidade o pH da solução extraída das amostras de terra da camada de 0,15 a 1,2 m, em comparação com a vegetação de Cerrado (LILIENFEIN et al., 2000). A acidificação do solo em áreas florestais pode ser relacionada com a lixiviação de bases ou pela absorção desses elementos pelas árvores, o que seria mais evidente em solos intemperizados, pobres em nutrientes e com baixo tamponamento. Parte da acidez do solo pode estar relacionada à liberação de ácidos orgânicos durante a decomposição da serapilheira, sendo normalmente observado maior pH em água na biomassa de plantas decíduas que coníferas (ALEXANDER e CRESSE, 1995).

Os teores de Al trocável variaram de 3,6 a 9,9 $\mathrm{cmol}_{\mathrm{c}}$ $\mathrm{dm}^{-3}$ (Tabela 2), com maiores valores no reflorestamento de pinus (P20) em todas as camadas, justificado pelos baixos valores de $\mathrm{pH}$, facilitando a solubilização do Al trocável no solo. Os menores valores foram observados no solo sob mata nativa. Os teores de Al trocável aumentaram em profundidade no $\mathrm{CN}$ e A18, o que poderia estar ligado ao efeito da matéria orgânica complexando parte desse elemento e reduzindo sua extração pela solução de KCl. Essa hipótese, entretanto, se confirmou apenas parcialmente pela análise de correlação entre $\mathrm{Al} \mathrm{e} \mathrm{C}_{\mathrm{Org}}$. $(\mathrm{r}=-0,24 * *$, Tabela 3$)$. Deve-se destacar que os altos teores de $\mathrm{Al}$ no solo são característicos desses solos com alto teor de matéria orgânica, conferindo elevado tamponamento de acidez.

O fósforo disponível do solo variou de 0,3 a 11,2 $\mathrm{mg} \mathrm{dm}^{-3}$, sem alteração em profundidade, com maiores teores no solo sob pinus com 20 anos de idade (P20) e no campo nativo, na camada de 0,0 a $0,05 \mathrm{~cm}$. Na média das profundidades, os maiores teores de fósforo foram constatados no P20 (Tabela 4). Essa variação pode estar associada à absorção diferenciada de $\mathrm{P}$ pelo pinus, ou à influência de fungos micorrízicos que ocorrem naturalmente na área e modificam a conformação do sistema radicular e a habilidade da planta em aproveitar esse elemento presente normalmente em baixos teores no solo. A variação na idade e diversidade da vegetação pode alterar a atividade micorrízica e os teores de nutrientes absorvidos pela planta (SIQUEIRA e FRANCO, 1988), mesmo considerando-se que não tenha sido feita a inoculação das mudas. Outro fator a considerar é a ciclagem desse elemento pela matéria orgânica, muito embora o coeficiente de correlação entre $\mathrm{P} \mathrm{e}_{\text {Org. }}$ tenha sido baixo $\left(\mathrm{r}=0,38^{* *}, \mathrm{p}<0,01\right.$, Tabela 3$)$.

Tabela 1 - Teores e estoques de carbono orgânico no solo em diferentes profundidades, no campo nativo (CN), reflorestamento de pinus com 12 anos (P12) e 20 anos (P20) de idade, reflorestamento de araucária com 18 anos (A18) de idade e mata nativa (MN), em Campo Belo do Sul, SC

Table 1 - Soil organic carbon contents and stocks in different depths, under native grassland (CN), loblolly pine (Pinus taeda) 12 -year-old (P12) and 20-year-old reforestation (P2O); 18-year-old araucaria (Araucaria angustifolia) reforestation (A18); and native forest (MN), in Campo Belo do Sul, SC, Brazil

\begin{tabular}{|c|c|c|c|c|c|c|c|}
\hline \multirow[t]{2}{*}{ Sistema } & \multicolumn{5}{|c|}{ Profundidade do Solo $(\mathrm{m})$} & \multicolumn{2}{|c|}{ Equação Ajustada para Profundidade } \\
\hline & $0,0-0,05$ & $0,5-0,1$ & $0,1-0,2$ & $0,2-0,4$ & Média & & \\
\hline & \multicolumn{7}{|c|}{ Teor de Carbono Orgânico, $\mathrm{g} \mathrm{kg}^{-1} \quad(\mathrm{CV}=26 \%)$} \\
\hline $\mathrm{CN}$ & $49,8 \mathrm{~b}$ & 37,2 a & 32,1 a & $27,8 \mathrm{~b}$ & $32,8 \mathrm{ab}$ & $\mathrm{Y}=54,5-7,12^{* *} \mathrm{X}$ & $\mathbf{R}^{2}=0,84$ \\
\hline P12 & $44,9 \mathrm{c}$ & 38,7 a & 32,7 a & $26,5 \mathrm{~b}$ & $31,9 \mathrm{bc}$ & $\mathrm{Y}=51,0-6,12 * * \mathrm{X}$ & $\mathrm{R}^{2}=0,81$ \\
\hline $\mathrm{P} 20$ & $46,1 \mathrm{c}$ & $37,1 \mathrm{a}$ & 35,2 a & $32,1 \mathrm{a}$ & 35,2 a & $\mathrm{Y}=48,6-4,39^{* *} \mathrm{X}$ & $\mathrm{R}^{2}=0,61$ \\
\hline A 18 & $39,6 \mathrm{~d}$ & $33,2 \mathrm{~b}$ & $29,2 \mathrm{~b}$ & $25,1 \mathrm{~b}$ & $28,9 \mathrm{c}$ & $Y=43,6-4,75^{* *} X$ & $\mathrm{R}^{2}=0,72$ \\
\hline \multirow[t]{3}{*}{ MN } & 55,8 a & $34,9 \mathrm{~b}$ & $27,1 \mathrm{~b}$ & $22,5 \mathrm{c}$ & $29,4 \mathrm{c}$ & $\mathrm{Y}=82,4-31,2^{* *} \mathrm{X}+4,09^{* *} \mathrm{X}^{2}$ & $\mathrm{R}^{2}=0,88$ \\
\hline & \multicolumn{7}{|c|}{ Estoque de Carbono Orgânico, $\mathrm{kg} \mathrm{m}^{-2}(\mathrm{CV}=50 \%)$} \\
\hline & $0,0-0,05$ & $0,5-0,1$ & $0,1-0,2$ & $0,2-0,4$ & & Soma & \\
\hline $\mathrm{CN}$ & $2,23 \mathrm{~b}$ & $1,82 \mathrm{a}$ & $3,33 \mathrm{ab}$ & 6,12 a & & 13,50 & \\
\hline $\mathrm{P} 12$ & $1,78 \mathrm{c}$ & $1,84 \mathrm{a}$ & $3,45 \mathrm{ab}$ & $5,78 \mathrm{~b}$ & & 12,85 & \\
\hline P20 & $2,05 \mathrm{~b}$ & $1,84 \mathrm{a}$ & 3,67 a & 6,58 a & & 14,15 & \\
\hline A18 & $2,04 \mathrm{~b}$ & $1,80 \mathrm{a}$ & $3,19 \mathrm{~b}$ & $5,47 \mathrm{~b}$ & & 12,50 & \\
\hline MN & $2,63 \mathrm{a}$ & $1,90 \mathrm{a}$ & $3,15 \mathrm{~b}$ & $5,33 \mathrm{~b}$ & & 13,07 & \\
\hline
\end{tabular}

Médias seguidas pela mesma letra na coluna não diferem entre si, pelo teste de Tukey a $5 \%$ de probabilidade. ${ }^{* *}$ Significativo a $1 \%$ de probabilidade; * 5 a $1 \%$ de probabilidade; e CV: coeficiente de variação.

R. Árvore, Viçosa-MG, v.32, n.2, p.217-224, 2008 
Tabela 2 - Acidez do solo expressa pelos valores de $\mathrm{pH}$ em água, $\mathrm{pH}$ em $\mathrm{CaCl}_{2}, \mathrm{pH} \mathrm{SMPe} \mathrm{Al}$ trocável do solo em diferentes profundidades, no campo nativo (CN), reflorestamento de pinus com 12 anos (P12) e 20 anos (P20) de idade, reflorestamento de araucária com 18 anos (A18) de idade e mata nativa (MN), em Campo Belo do Sul, SC

Table 2 - Soil acidity expressed by $\mathrm{pH}$ water, $\mathrm{pH} \mathrm{CaCl}, \mathrm{pH} \mathrm{SMP}$ and soil exchangeable Al, at different depths, under native grassland (CN), loblolly pine (Pinus taeda) 12-year-old (P12) and 20-year-old (P2O) reforestation; 18-yearold araucaria (Araucaria angustifolia) reforestation (A18); and native forest (MN), in Campo Belo do Sul, SC, Brazil

\begin{tabular}{|c|c|c|c|c|c|c|c|}
\hline \multirow[t]{2}{*}{ Sistema } & \multicolumn{5}{|c|}{ Profundidade do Solo (m) } & \multicolumn{2}{|l|}{ Equação Ajustada para Profundidade } \\
\hline & $0,0-0,05$ & $0,5-0,1$ & $0,1-0,2$ & $0,2-0,4$ & Média & & \\
\hline \multicolumn{8}{|c|}{ pH Água $(\mathrm{CV}=6 \%)$} \\
\hline $\mathrm{CN}$ & $5,12 \mathrm{~b}$ & 5,09 a & 5,09 a & $5,04 \mathrm{a}$ & 5,07 a & $\mathrm{ns}$ & \\
\hline $\mathrm{P} 12$ & $4,65 \mathrm{~d}$ & $4,80 \mathrm{~b}$ & $4,81 \mathrm{~b}$ & $4,88 \mathrm{~b}$ & $4,82 \mathrm{~b}$ & $\mathrm{~ns}$ & \\
\hline P20 & $4,56 \mathrm{~d}$ & $4,60 \mathrm{c}$ & $4,57 \mathrm{c}$ & $4,52 \mathrm{c}$ & $4,55 \mathrm{c}$ & $\mathrm{ns}$ & \\
\hline A 18 & $5,33 \mathrm{a}$ & 5,09 a & $5,10 \mathrm{a}$ & 5,09 a & $5,12 \mathrm{a}$ & $\mathrm{ns}$ & \\
\hline MN & $4,96 \mathrm{c}$ & $4,94 \mathrm{a}$ & $4,87 \mathrm{~b}$ & $4,83 \mathrm{~b}$ & $4,87 \mathrm{~b}$ & $\mathrm{~ns}$ & \\
\hline \multicolumn{8}{|c|}{$\mathrm{pH} \mathrm{CaCl}{ }_{2} 0,01 \mathrm{~mol} \mathrm{~L}^{-1}(\mathrm{CV}=4 \%)$} \\
\hline $\mathrm{CN}$ & $4,23 \mathrm{a}$ & $4,05 \mathrm{a}$ & 4,06 a & 4,05 a & 4,07 a & $Y=4,22-0,05^{*} \quad X$ & $\mathrm{R}^{2}=0,14$ \\
\hline $\mathrm{P} 12$ & $3,89 \mathrm{~b}$ & $4,01 \mathrm{ab}$ & 4,08 a & 4,08 a & $4,05 \mathrm{a}$ & $\mathrm{Y}=3,86+0,06^{* *} \mathrm{X}$ & $\mathrm{R}^{2}=0,35$ \\
\hline $\mathrm{P} 20$ & $3,86 \mathrm{~b}$ & $3,91 \mathrm{~b}$ & $3,90 \mathrm{~b}$ & $3,91 \mathrm{~b}$ & $3,90 \mathrm{~b}$ & $\mathrm{~ns}$ & \\
\hline A 18 & 4,28 a & $4,10 \mathrm{a}$ & 4,05 a & $4,01 \mathrm{a}$ & $4,07 \mathrm{a}$ & $\mathrm{Y}=4,32-0,08 * * \mathrm{X}$ & $\mathrm{R}^{2}=0,30$ \\
\hline $\mathrm{MN}$ & 4,24 a & 4,09 a & 4,01 a & 3,99 a & $4,04 \mathrm{a}$ & $\mathrm{ns}$ & \\
\hline \multicolumn{8}{|c|}{ pH SMP $(\mathrm{CV}=6 \%)$} \\
\hline $\mathrm{CN}$ & 5,73 a & 5,64 a & 5,64 a & $5,71 \mathrm{a}$ & 5,69 a & $\mathrm{ns}$ & \\
\hline $\mathrm{P} 12$ & $5,34 \mathrm{~b}$ & $5,45 \mathrm{~b}$ & $5,43 \mathrm{~b}$ & $5,54 \mathrm{~b}$ & $5,48 \mathrm{~b}$ & $\mathrm{~ns}$ & \\
\hline P20 & $4,98 \mathrm{c}$ & $5,06 \mathrm{c}$ & $4,98 \mathrm{c}$ & $5,10 \mathrm{c}$ & $5,05 \mathrm{c}$ & $\mathrm{ns}$ & \\
\hline A 18 & 5,82 a & $5,54 \mathrm{ab}$ & 5,63 a & $5,70 \mathrm{a}$ & 5,67 a & $\mathrm{ns}$ & \\
\hline $\mathrm{MN}$ & 5,69 a & 5,62 a & 5,63 a & $5,53 \mathrm{~b}$ & $5,59 \mathrm{ab}$ & $\mathrm{ns}$ & \\
\hline \multicolumn{8}{|c|}{ Al trocável, $\mathrm{cmol}_{\mathrm{c}} \mathrm{dm}^{-3}(\mathrm{CV}=28 \%)$} \\
\hline $\mathrm{CN}$ & $5,69 \mathrm{~b}$ & $7,50 \mathrm{~b}$ & $8,72 \mathrm{~b}$ & $8,72 \mathrm{~b}$ & $8,19 \mathrm{~b}$ & $\mathrm{Y}=2,81+3,30 * * \mathrm{X}-0,45 * * \mathrm{X}^{2}$ & $\mathrm{R}^{2}=0,71$ \\
\hline $\mathrm{P} 12$ & 8,88 a & 9,39 a & $8,61 \mathrm{~b}$ & $8,22 \mathrm{~b}$ & $8,55 \mathrm{~b}$ & $\mathrm{~ns}$ & \\
\hline $\mathrm{P} 20$ & 9,38 a & 9,55 a & 9,89 a & $10,11 \mathrm{a}$ & 9,89 a & ns & \\
\hline A 18 & 3,56 a & $5,30 \mathrm{c}$ & $6,86 \mathrm{c}$ & $7,97 \mathrm{bc}$ & $6,81 \mathrm{c}$ & $\mathrm{Y}=2,23+1,48 * * \mathrm{X}$ & $\mathrm{R}^{2}=0,51$ \\
\hline MN & $5,05 \mathrm{~b}$ & $7,55 \mathrm{~b}$ & $8,33 \mathrm{~b}$ & $8,61 \mathrm{~b}$ & $7,96 \mathrm{~b}$ & $\mathrm{Y}=4,52+1,15 * * \mathrm{X}$ & $\mathrm{R}^{2}=0,32$ \\
\hline
\end{tabular}

Médias seguidas pela mesma letra na coluna não diferem entre si, pelo teste de Tukey a $5 \%$ de probabilidade. ${ }^{* *}$ Significativo a $1 \%$ de probabilidade; ${ }^{*} 5$ a $1 \%$ de probabilidade; ${ }^{\text {ns }}$ não-significativo a $5 \%$ de probabilidade; e CV: coeficiente de variação.

Tabela 3 - Coeficientes de correlação de Pearson entre as variáveis analisadas, em todas as camadas de solo, nas áreas florestais e de campo nativo analisadas em conjunto, em Campo Belo do Sul, SC ( $\mathrm{n}=160)$

Table 3 - Pearson correlation coefficients between analysed variables considering all soil layers, including forested and native grassland areas, in Campo Belo do Sul, SC, Brazil $(n=160)$

\begin{tabular}{|c|c|c|c|c|c|c|c|c|}
\hline Variáveis & $\mathrm{pH}_{\mathrm{H} 2 \mathrm{O}}$ & $\mathrm{pH}_{\mathrm{CaCl} 2}$ & $\mathrm{pH}_{\mathrm{SMP}}$ & $\mathrm{P}$ & $\mathrm{K}$ & $\mathrm{Ca}$ & $\mathrm{Mg}$ & $\mathrm{Al}$ \\
\hline $\mathrm{C}_{\text {Org. }}$ & $-0,06^{\mathrm{ns}}$ & $0,16^{*}$ & $-0,10^{\mathrm{ns}}$ & $0,38^{* * *}$ & $0,50^{* *}$ & $0,33^{* *}$ & $0,16^{*}$ & $-0,24^{* * *}$ \\
\hline $\mathrm{pH}_{\mathrm{H} 2 \mathrm{O}}$ & & $0,71^{* * *}$ & $0,74^{* *}$ & $-0,17^{*}$ & $0,32^{* *}$ & $0,33^{* *}$ & $0,40^{* *}$ & $-0,46^{* *}$ \\
\hline $\mathrm{pH}_{\mathrm{CaCl} 2}$ & & & $0,70^{* *}$ & $-0,08^{\mathrm{ns}}$ & $0,35^{* *}$ & $0,35^{* *}$ & $0,41^{* *}$ & $-0,53^{* *}$ \\
\hline $\mathrm{pH}_{\mathrm{SMP}}$ & & & & $-0,28^{* *}$ & $0,27^{* *}$ & $0,29^{* * *}$ & $0,35^{* *}$ & $-0,48^{* * *}$ \\
\hline $\mathrm{P}$ & & & & & $0,37^{* *}$ & $0,13^{\mathrm{ns}}$ & $0,01^{\mathrm{ns}}$ & $-0,05^{\mathrm{ns}}$ \\
\hline K & & & & & & $0,29^{\text {** }}$ & $0,47^{* *}$ & $-0,39^{* * *}$ \\
\hline $\mathrm{Ca}$ & & & & & & & $0,56^{* *}$ & $-0,78^{* * *}$ \\
\hline $\mathrm{Mg}$ & & & & & & & & $-0,54^{* *}$ \\
\hline
\end{tabular}

$\mathbf{C}_{\text {Org }}=$ carbono orgânico total; $\mathbf{P}=\mathrm{P}$ extraível; $\mathbf{K}=\mathrm{K}$ trocável; $\mathbf{C a}=\mathrm{Ca}$ trocável; $\mathbf{M g}=\mathrm{Mg}$ trocável; e $\mathbf{A l}=\mathrm{Al}$ trocável. ${ }^{* *}$ Significativo $\mathbf{C}_{\text {Org }}=$ carbono organico total; $\mathbf{P}=\mathrm{P}$ extraível; $\mathbf{K}=\mathrm{K}$ trocável; $\mathbf{C a}=$ Ca trocável; $\mathbf{M g}=\mathrm{Mg}$ trocá
a $1 \%$ de probabilidade; 5 a $1 \%$ de probabilidade; e ${ }^{\text {ns }}$ não-significativo a $5 \%$ de probabilidade. 
Tabela 4 - Teores de Pextraível e K, Ca, e Mg trocáveis do solo em diferentes profundidades, no campo nativo (CN), reflorestamento de pinus com 12 anos (P12) e 20 anos (P20) de idade, reflorestamento de araucária com 18 anos (A18) de idade e mata nativa (MN), em Campo Belo do Sul, SC

Table 4 - Soil available $P$ and soil exchangeable $K$, Ca e $\mathrm{Mg}$, in different depths, under native grassland (CN), loblolly pine (Pinus taeda) 12-year-old (P12) and 20-year-old (P2O) reforestation; 18-year-old araucaria (Araucaria angustifolia) reforestation (A18); and native forest (MN), in Campo Belo do Sul, SC, Brazil

\begin{tabular}{|c|c|c|c|c|c|c|c|}
\hline \multirow[t]{2}{*}{ Sistema } & \multicolumn{5}{|c|}{ Profundidade do Solo (m) } & \multicolumn{2}{|l|}{ Equação Ajustada para Profundidade } \\
\hline & $0,0-0,05$ & $0,5-0,1$ & $0,1-0,2$ & $0,2-0,4$ & Média & & \\
\hline \multicolumn{8}{|c|}{ P disponível (Mehlich-1) $\quad(\mathrm{CV}=81 \%)$} \\
\hline $\mathrm{CN}$ & $8,98 \mathrm{~b}$ & $6,39 \mathrm{~b}$ & $3,06 \mathrm{ab}$ & $3,43 \mathrm{~b}$ & $4,40 \mathrm{~b}$ & ns & \\
\hline $\mathrm{P} 12$ & $4,20 \mathrm{c}$ & $3,96 \mathrm{~b}$ & $2,10 \mathrm{~b}$ & $0,25 \mathrm{c}$ & $1,67 \mathrm{c}$ & ns & \\
\hline P20 & $11,20 \mathrm{a}$ & 10,16 a & 5,88 a & $6,52 \mathrm{a}$ & $7,40 \mathrm{a}$ & $\mathrm{ns}$ & \\
\hline A 18 & $6,42 \mathrm{bc}$ & $2,56 \mathrm{~b}$ & $3,69 \mathrm{ab}$ & $2,98 \mathrm{bc}$ & $3,53 \mathrm{~b}$ & $\mathrm{~ns}$ & \\
\hline $\mathrm{MN}$ & $6,91 \mathrm{bc}$ & $4,21 \mathrm{~b}$ & $4,11 \mathrm{ab}$ & $2,27 \mathrm{bc}$ & $3,55 \mathrm{~b}$ & $\mathrm{~ns}$ & \\
\hline \multicolumn{8}{|c|}{$\mathrm{K}$ trocável (Mehlich-1) $(\mathrm{CV}=75 \%)$} \\
\hline $\mathrm{CN}$ & $0,40 \mathrm{a}$ & $0,19 \mathrm{~ns}$ & $0,12 \mathrm{a}$ & 0,09 a & 0,15 a & $\mathrm{Y}=0,673-0,325^{* *} \mathrm{X}+0,045^{* *} \mathrm{X}^{2}$ & $\mathrm{R}^{2}=0,80$ \\
\hline P12 & $0,09 \mathrm{c}$ & 0,07 & $0,05 \mathrm{~b}$ & $0,04 \mathrm{~b}$ & $0,05 \mathrm{c}$ & $\mathrm{Y}=0,102-0,016^{* *} \mathrm{X}$ & $\mathrm{R}^{2}=0,61$ \\
\hline P20 & $0,11 \mathrm{c}$ & 0,09 & $0,09 \mathrm{~b}$ & $0,07 \mathrm{ab}$ & $0,08 \mathrm{~b}$ & $\mathrm{Y}=0,117-0,012 * * \mathrm{X}$ & $\mathrm{R}^{2}=0,55$ \\
\hline A18 & $0,16 \mathrm{~b}$ & 0,11 & $0,11 \mathrm{ab}$ & $0,07 \mathrm{ab}$ & $0,09 \mathrm{~b}$ & $\mathrm{Y}=0,187-0,030 * * \mathrm{X}$ & $\mathrm{R}^{2}=0,41$ \\
\hline $\mathrm{MN}$ & $0,16 \mathrm{~b}$ & 0,10 & $0,07 \mathrm{~b}$ & $0,05 \mathrm{~b}$ & $0,08 \mathrm{~b}$ & $\mathrm{Y}=0,184-0,035^{* *} \mathrm{X}$ & $\mathrm{R}^{2}=0,64$ \\
\hline \multicolumn{8}{|c|}{ Ca trocável (Extrator $\left.\mathrm{KCl} 1,0 \mathrm{~mol} \mathrm{~L}^{-1}\right) \quad(\mathrm{CV}=130 \%)$} \\
\hline $\mathrm{CN}$ & $1,62 \mathrm{c}$ & $0,80 \mathrm{~b}$ & $0,41 \mathrm{~b}$ & $0,27 \mathrm{ab}$ & $0,54 \mathrm{c}$ & $\mathrm{Y}=2,75-1,31 * * \mathrm{X}+0,17 * * \mathrm{X}^{2}$ & $\mathbf{R}^{2}=0,85$ \\
\hline $\mathrm{P} 12$ & $0,86 \mathrm{~d}$ & $0,39 \mathrm{c}$ & $0,24 \mathrm{~b}$ & $0,12 \mathrm{~b}$ & $0,28 \mathrm{c}$ & $\mathrm{Y}=0,99-0,24 * * \mathrm{X}$ & $\mathrm{R}^{2}=0,38$ \\
\hline $\mathrm{P} 20$ & $0,98 \mathrm{~cd}$ & $0,42 \mathrm{c}$ & $0,32 \mathrm{~b}$ & $0,24 \mathrm{~b}$ & $0,37 \mathrm{c}$ & $\mathrm{Y}=1,07-0,23 * * \mathrm{X}$ & $\mathrm{R}^{2}=0,35$ \\
\hline A 18 & 5,36 a & $2,52 \mathrm{a}$ & $1,67 \mathrm{a}$ & $1,05 \mathrm{a}$ & 1,93 a & $\mathrm{Y}=6,09-1,38 * * \mathrm{X}$ & $\mathrm{R}^{2}=0,45$ \\
\hline $\mathrm{MN}$ & $3,32 \mathrm{~b}$ & $1,32 \mathrm{~b}$ & $0,96 \mathrm{ab}$ & $0,44 \mathrm{ab}$ & $1,04 \mathrm{~b}$ & $\mathrm{Y}=3,76-0,90 * * \mathrm{X}$ & $\mathrm{R}^{2}=0,53$ \\
\hline \multicolumn{8}{|c|}{ Mg trocável (Extrator $\left.\mathrm{KCl} 1,0 \mathrm{~mol} \mathrm{~L}^{-1}\right) \quad(\mathrm{CV}=84 \%)$} \\
\hline $\mathrm{CN}$ & $1,42 \mathrm{~b}$ & $0,15 \mathrm{c}$ & 0,72 a & $0,26 \mathrm{~b}$ & $0,51 \mathrm{cb}$ & $\mathrm{Y}=2,39-1,31 * * \mathrm{X}+0,20 * * \mathrm{X}^{2}$ & $\mathbf{R}^{2}=0,50$ \\
\hline $\mathrm{P} 12$ & $0,15 \mathrm{~d}$ & $0,42 \mathrm{~b}$ & $0,33 \mathrm{~b}$ & $0,12 \mathrm{~b}$ & $0,21 \mathrm{~d}$ & $\mathrm{Y}=0,31-0,59 * * \mathrm{X}-0,12 * * \mathrm{X}^{2}$ & $\mathrm{R}^{2}=0,26$ \\
\hline $\mathrm{P} 20$ & $0,27 \mathrm{~d}$ & $0,30 \mathrm{bc}$ & $0,45 \mathrm{~b}$ & $0,35 \mathrm{ab}$ & $0,36 \mathrm{~cd}$ & ns & \\
\hline A 18 & $1,64 \mathrm{a}$ & $1,04 \mathrm{a}$ & 0,83 a & $0,57 \mathrm{a}$ & $0,83 \mathrm{a}$ & $\mathrm{Y}=1,87-0,34 * * \mathrm{X}$ & $\mathbf{R}^{2}=0,46$ \\
\hline $\mathrm{MN}$ & $0,72 \mathrm{c}$ & $0,37 \mathrm{bc}$ & $0,79 \mathrm{a}$ & $0,56 \mathrm{a}$ & $0,62 \mathrm{~b}$ & ns & \\
\hline
\end{tabular}

Médias seguidas pela mesma letra na coluna não diferem entre si, pelo teste de Tukey a $5 \%$ de probabilidade. ${ }^{* *}$ Significativo a $1 \%$ de probabilidade; * 5 a $1 \%$ de probabilidade; ${ }^{n s}$ não-significativo a $5 \%$ de probabilidade; e CV: coeficiente de variação.

Os teores de K trocável do solo decresceram em profundidade, com maior concentração na média das profundidades, verificada no campo nativo, e o menor teor ocorreu no solo sob pinus com 12 anos (Tabela 4). Os teores mais elevados de $\mathrm{K}$ na camada superficial do solo apontam para ciclagem pela fitomassa, especialmente no caso do campo nativo, em que se realiza uma roçada anual para controle da altura das plantas. Em estudo realizado na região de Viçosa, $\mathrm{MG}$, observou-se maior teor de $\mathrm{K}$ em solo sob vegetação de gramíneas, em comparação com diferentes coberturas arbóreas (CÓSER et al., 1990). No reflorestamento com pinus se observou menor teor de K na camada superficial $(0,0-0,05 \mathrm{~cm})$, o que seria advindo da sua elevada capacidade de absorção desse elemento. Ainterdependência entre teores de potássio e os constituintes orgânicos nos solos estudados foi evidenciado pelo coeficiente de correlação de 0,50 (p $=0,01$ ) entre $\mathrm{Ke} \mathrm{C}_{\text {Org. }}$ (Tabela 3 ).
Os teores de Ca trocável no solo variaram de 0,1 a $5,4 \mathrm{cmol}_{\mathrm{c}} \mathrm{dm}^{-3}$, com maiores valores no reflorestamento de araucária e mata nativa em todas as camadas (Tabela 4). Em profundidade houve diminuição nos teores desse elemento, com ajustes quadrático no $\mathrm{CN}$ e linear nos demais tratamentos, os quais apresentaram coeficientes de determinação baixos $\left(\mathrm{R}^{2}<0,53\right)$. Com $\mathrm{Mg}$ trocável, os teores no solo foram em média mais altos sob reflorestamento de araucária. Não houve variação na concentração do elemento em profundidade no P20 e na MN, com decréscimo em direção à subsuperfície nos demais sistemas, mas igualmente com baixo $\mathrm{R}^{2}$ (Tabela 4). Os teores de $\mathrm{Mg}$ e Ca trocável no solo sob reflorestamento de araucária foram influenciados pela calagem efetuada na sua implantação.

As relações entre os sistemas florestais e a composição química do solo anteriormente apresentadas são, em grande parte, corroboradas por Silva Júnior 
et al. (1987), que avaliaram duas espécies arbóreas folhosas e Pinus elliotti em Viçosa, MG, na camada de 0,0 a 0,2 cm de profundidade, 19 anos após o plantio das árvores. Esses autores constataram maior retorno de nutrientes ao solo por unidade de peso de matéria seca nas parcelas com folhosas em relação às coníferas. Além disso, os teores de Ca e Mg no solo sob folhosas foram cinco vezes superiores ao do solo sob pinus, concordando, porém em menor magnitude, com o apresentado na Tabela 4. Observaram também maior teor de $\mathrm{Al}$ e menor de $\mathrm{K}$ no solo sob pinus em relação às demais plantas, à semelhança do apresentado nas Tabelas 2 e 4 . Esses autores justificaram tais resultados em função da absorção diferenciada de nutrientes pelas árvores, além de variações na lixiviação e velocidade de decomposição da serapilheira.

A contribuição da vegetação em relação aos nutrientes no solo deve ser considerada especialmente quando as áreas florestais são submetidas à exploração. A remoção de nutrientes que ocorre nos produtos florestais é dependente da espécie, idade de corte, densidade de árvores e biomassa total produzida (VALERI et al., 1989). Destaca-se que, juntamente com os nutrientes, também o carbono é removido, podendo influenciar a matéria orgânica do solo. Essa preocupação é relevante na região de Lages, em virtude do aproveitamento inclusive dos resíduos da floresta para produção de energia, ocasionando maior extração de nutrientes. Tal prática implicaria cuidado adicional no planejamento do manejo florestal em termos de garantia das condições de fertilidade do solo, especialmente nesse ambiente com baixa reserva de nutrientes, assegurando-se o desenvolvimento vegetal e a produtividade da floresta (CHAVES e CORRÊA, 2005).

\section{CONCLUSÕES}

Os reflorestamentos com pinus e araucária mantiveram os estoques de $\mathrm{C}$ orgânico na camada do solo de 0,0-0,4 $\mathrm{m}$ em níveis equivalentes aos de mata e campo.

A acidez do solo e os teores de $\mathrm{P}$ disponível foram maiores, na média das camadas analisadas, no reflorestamento de pinus com 20 anos de idade.

Entre os nutrientes, verificaram-se teores baixos a médios de fósforo disponível e de potássio, cálcio e magnésio trocável.

\section{AGRADECIMENTOS}

À Florestal Gateados, pelo apoio nos trabalhos de campo, em especial ao Sr. Valdir Diehl Ribeiro; e ao acadêmico João Carlos Medeiros, pelo auxílio nas análises de laboratório.

\section{REFERÊNCIAS}

ALEXANDER, C. E.; CRESSE, M. S. An assessment of the possible impact of expansion of native woodland cover on the chemistry of Scottish freshwaters. Forest Ecology and Management, v.73, n.1, p.1-27, 1995.

BALBINOT, R. et al. Inventário do carbono orgânico em um plantio de Pinus taeda aos 5 anos de idade no Rio Grande do Sul. Revista Ciências Exatas e Naturais, v.5, n.1, p.59-68, 2003.

BROWN, S. et al. Soil biological processes in tropical ecossystems. In: WOOMER, P. L.; SWIFT, M. J. (Eds.) The biological management of tropical soil fertility. Chichester: John Wiley \& Sons, 1994. p.15-46.

CHAVES, R. Q.; CORRÊA, G. F. Macronutrientes no sistema solo-Pinus caribaea Morelet em plantios apresentando amarelecimento das acículas e morte de plantas. Revista Árvore, v.29, p.691-700, 2005.

CÓSER, A. C. et al. Efeito de diferentes coberturas vegetais sobre as características físicas e químicas de um Latossolo Vermelho-Amarelo álico. Revista Ceres, v.37, n.210, p.167-176, 1990.

FEARNSIDE, P. M. Uncertainty in land-use change and forestry sector mitigation options for global warming: Plantation silviculture versus avoided deforestation. Biomass and Bioenergy, v.18, n.6, p.457-468, 2000.

FEISTAUER, D. et al. Seqüestro de carbono de um sistema agroflorestal com bracatinga (Mimosa scabrella Bentham) na região metropolitana de Curitiba, PR. In: CONGRESSO BRASILEIRO DE SISTEMAS AGROFLORESTAIS, 5., 2004, Curitiba. Anais... Curitiba: Embrapa Florestas, 2004. p.175-177. (Documentos, 98)

FERNANDES, A. V.; BACKES, A. Produtividade primária em floresta com Araucaria angustifolia no Rio Grande do Sul. Iheringia, Série Botânica, v.50, n.1, p.63-78, 1998. 
FERRAZ, C.; MOTTA, R. S. Exploração florestal, sustentabilidade e o mecanismo de desenvolvimento limpo. Ciência e Ambiente, n.20, p.83-98, 2000.

FIGUEIREDO FILHO, A. et al. Avaliação estacional da deposição de serapilheira em uma Floresta Ombrófila Mista localizada no sul do estado do Paraná. Ciência Florestal, v.13, n.1, p.11-18, 2003.

HAAG, H. P. Ciclagem de nutrientes em florestas tropicais. Campinas: Fundação Cargill, 1985. 144p.

LILIENFEIN, J. et al. Soil acidification in Pinus caribaea forests on Brazilian savanna Oxisols. Forest Ecology and Management, v.128, n.1, p.145-157, 2000.

LIMA, V. C. et al. Efeito da substituição de campo natural por Pinus na dinâmica do carbono do solo. Revista do Setor de Ciências Agrárias, v.14, n.1, p.7-12, 1995.

MAINARDI, G. L.; SCHNEIDER, P. R.; FINGER, C. A. G. Produção de Pinus taeda L. na região de Cambará do Sul, RS. Ciência Florestal, v.6, n.1, p.39-52, 1996.

PAIXÃO, F. A. et al. Quantificação do estoque de carbono e avaliação econômica de diferentes alternativas de manejo em um plantio de eucalipto. Revista Árvore, v.30, n.3, p.411-420, 2006.

SANTA CATARINA. Gabinete de Planejamento e Coordenação Geral. Sub-chefia de Estatística, Geografia e Informática. Atlas de Santa Catarina. Rio de Janeiro: Aerofoto Cruzeiro, 1986. 176p.
SHAPIRO, S. S.; WILK, M. B. An analysis of variance test for normality. Biometrika, v.52, n.3-4, p.591-611, 1965.

SILVA, L. F. Alterações edáficas provocadas por essências florestais implantadas em solos de tabuleiro no Sul da Bahia. Revista

Theobroma, v.18, n.4, p.259-267, 1988.

SILVA JÚNIOR, M. C.; BARROS, N. F.; PINHEIRO, A. L. Concentração de nutrientes e efeito sobre a fertilidade do solo de três espécies florestais na região de Viçosa, MG. Brasil Florestal, v.19, n.1, p.43-48, 1987.

SIQUEIRA, J.O.; FRANCO, A.A. Biotecnologia do solo: fundamentos e perspectivas. Brasília: MEC/ABEAS/ESAL/FAEPE, 1988. 236p.

TEDESCO, M. J. et al. Análises de solo, plantas e outros materiais. Porto Alegre: Universidade Federal do Rio Grande do Sul, 1995. $174 \mathrm{p}$.

TREVISAN, E. et al. Morfologia dos horizontes orgânicos acumulados sob povoamento de Pinus taeda L., em três sítios distintos.

Revista do Setor de Ciências Agrárias, v.9, n.1, p.59-62, 1987.

VALERI, S. V.; REISSMANN, C. B.; SANTOS FILHO, A. Exportação de nutrientes de povoamentos de Pinus taeda L. desbastados em diferentes idades. Revista Floresta, v.19, n.1, p.62-68, 1989.

WISNIEWSKI, C.; REISSMANN, C. B. Deposição de serapilheira e de nutrientes em plantios de Pinus taeda L. na região de Ponta Grossa, PR. Arquivos de Biologia e Tecnologia, v.39, n.2, p.435-442, 1996. 